\title{
A descriptive study of transfusion dependent anaemias in paediatric unit, General Hospital, Ampara
}

\author{
M I Rifaya ${ }^{1}$ \\ Sri Lanka Journal of Child Health, 2011; 40: 63 \\ (Key words: Thalassaemia; children; Ampara)

\section{Background}

General Hospital Ampara is a tertiary care centre where a considerable number of children are admitted to the paediatric unit every month for management of transfusion dependent anaemias.

\section{Objective}

To study socio-demographic and clinical aspects of transfusion dependent anaemias in the paediatric unit, General Hospital Ampara

\section{Method}

An observational descriptive study was carried out from July to December 2008 on children admitted to the paediatric unit, General Hospital, Ampara for management of transfusion dependent anaemias. Information on socio-demographic data, treatment modalities and complications was gathered using questionnaires.

\section{Results}

The total number of children was 67. Age groups of the children were: 6 months to 1 year $(1.4 \%), 2-5$ years $(31 \%), 6-10$ years $(42 \%)$ and $11-15$ years $(25 \%)$. Fifty five percent were girls. Ninety five percent of children were from the Eastern province $14 \%$ of whom were from the Ampara district. Eighty two percent had thalassaemia major, 5\% thalassaemia minor, $4.4 \% \quad \beta$-thalassaemia/HbE, $3 \%$ congenital dyserythropoietic anaemia and $1.4 \%$ sideroblastic anaemia. The diagnosis was not confirmed in $4.4 \%$. In $13 \%$ the parents were consanguineous and $15 \%$ had a sibling with a similar disease. In $40 \%$ one of the parents was a carrier. However, in $56 \%$ parents were not screened.

\footnotetext{
${ }^{1}$ Acting paediatrician, General Hospital Ampara

(Received on $6^{\text {th }}$ January 2010: Accepted on 19 February 2010)
}

With regard to treatment, $70 \%$ were on subcutaneous desferrioxamine and $20 \%$ on a combination of desferrioxamine and deferiprone. The oral deferiprone therapy was introduced for the first time in June 2008. Compliance was poor in $85 \%$; this was attributed to non availability of an adequate supply of subcutaneous desferrioxamine. Only 48\% had infusion pumps. In $41 \%$ the serum ferritin was above $3000 \mu \mathrm{g} / \mathrm{l}$ and of them $25 \%$ needed intravenous desferrioxamine therapy to bring down the serum ferritin level. Nineteen percent were splenectomised.

Regarding complications, $12 \%$ had cardiomyopathy, $6 \%$ hypothyroidism, $3 \%$ diabetes and in $33 \%$ the height was less than the $3^{\text {rd }}$ percentile.

In $80 \%$ father was the breadwinner of the family and in $9 \%$ both parents contributed financially. Monthly income in $65 \%$ was less than 10,000 rupees. Only $50 \%$ of parents had an adequate knowledge about thalassaemia regarding inheritance, complications and prevention.

\section{Conclusions and Recommendations}

- This study shows that transfusion related anaemias are prevalent among the paediatric population in Ampara.

- The importance of carrier screening and the parental knowledge on thalassaemia needs to be addressed as most of the children are from the rural area with poor socioeconomic backgrounds and consanguineous marriages are still widespread in the rural area.

- The compliance can be improved by providing infusion pumps through government funding and making the parents aware of the benefit of oral chelation therapy.

\section{Acknowledgements}

I thank all the paediatric intern medical officers of the Ampara General Hospital for their invaluable help. 